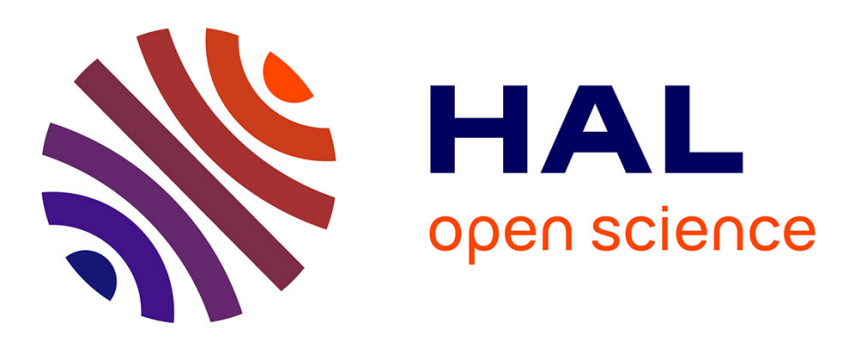

\title{
Laser-scanned tree stem filtering for forest inventories measurements
}

\author{
Joris Ravaglia, Alexandra Bac, Alexandre Piboule
}

\section{To cite this version:}

Joris Ravaglia, Alexandra Bac, Alexandre Piboule. Laser-scanned tree stem filtering for forest inventories measurements. Digital Heritage, Oct 2013, Marseille, France. pp.649 - 652, 10.1109/DigitalHeritage.2013.6743809 . hal-01376465

\section{HAL Id: hal-01376465 \\ https://hal.science/hal-01376465}

Submitted on 4 Oct 2016

HAL is a multi-disciplinary open access archive for the deposit and dissemination of scientific research documents, whether they are published or not. The documents may come from teaching and research institutions in France or abroad, or from public or private research centers.
L'archive ouverte pluridisciplinaire HAL, est destinée au dépôt et à la diffusion de documents scientifiques de niveau recherche, publiés ou non, émanant des établissements d'enseignement et de recherche français ou étrangers, des laboratoires publics ou privés. 


\section{Laser-Scanned Tree Stem Filtering for Forest Inventories Measurements}

\author{
Joris Ravaglia \\ Laboratoire des Sciences \\ de l'Information et des Systèmes \\ Université d'Aix-Marseille \\ Laboratoire de géomatique appliquée \\ Université de Sherbrooke \\ Sherbrooke, Canada \\ Email: joris.ravaglia@usherbrooke.ca
}

\author{
Bac Alexandra \\ Laboratoire des Sciences \\ de l'Information et des Systèmes \\ Université d'Aix-Marseille \\ Marseille, France \\ Email: alexandra.bac@univ-amu.fr
}

\author{
Alexandre Piboule \\ Office National des Forêts \\ Département Recherche et Développement \\ Nancy, France \\ Email: alexandre.piboule@onf.fr
}

\begin{abstract}
With specific flora and fauna, regional landscapes and forests constitute an important part of the cultural heritage. Several natural environments have already been classified as national or regional parks. The UNESCO World Heritage covers $13 \%$ of the protected forests in the world. Thus, preserving those sites represents a crucial issue. Such a safeguarding involves a detailed knowledge of the sites and forestry management plans. The management of a natural forest is traditionally based on forest plot inventories in which several features of the trees are measured. The set of data collected during these inventories represents the starting point of forest monitoring, flora preservation and risks prevention. Traditionally, measurements are made manually by operators. However, during the last decade, terrestrial laser scanning has become a new and promising way of measuring such attributes. This instrument provides a fine three dimensional point cloud virtual representation of the scanned scene. Trees location, stem diameter, and stem taper can be extracted from these point clouds using pattern recognition algorithms. In this paper we present a novel two steps way to improve the quality of tree branching detection in a three dimensional point cloud acquired by terrestrial laser scanner. This method was developped in order to enhance the results of a previous study. Our approach is based on the combination of a simplification step (using particle simulation), followed by a shape detection (discrete arcs of circle detection). It identifies the lack of accuracy in tree stem diameter measurements at branching junctions for further more detailled analysis.
\end{abstract}

\section{INTRODUCTION}

Forests and wooded areas are part of the national and regional cultural heritages because of their impact on ecological, societal and economical assets. One of the specificities of forests with respect to their contribution to the natural heritage lies in their long renewal and evolution cycle as well as in their lively and dynamic nature. As a consequence, a quantitative and regular monitoring of this evolution is necessary for the knowledge, the preservation and the promotion of this heritage. This is true not only for preservation spaces such as natural parks or wilderness areas, but also for managed forests (as shown by the "Forêt d'exception" label created by the ONF in 2007 or the numerous wooded areas classified by the UNESCO as world heritage).

Trees constitute an important factor of forests development and play a major role in environment preservation. Thus, they are given a particular attention. Many studies are related to forest inventories which are essential to manage and preserve forest environment. Traditional inventories consist in manually measuring several attributes of trees such as diameter at breast height (DBH, diameter of the trunk $1.30 \mathrm{~m}$ above the ground) or tree height.

Recently, terrestrial laser scanning has proven to be a promising tool for forest inventories measurements ([1], [2]). This instrument provides a three dimensional point cloud of the scene and allows automatic treatments as well as temporal follow-up of forest inventory plots. From these data, the position, DBH and stem taper of the scanned tree can be estimated ([3], [4]). This also permits the extraction of branching attributes of the trees ([5]) as well as biomass measurements ([6]).

Lately, Othmani et al. developed an efficient method to automatically extract the location of trees, their DBH and stem taper ([7]). However, this methodology may lead to inaccurate results at branches junctions. In this paper we propose a two steps enhancement of this methodology to improve measurement accuracy at these points. Our approach is based on the combination of a point cloud simplification and the introduction of a new tree cross-section model (discrete arcs of circle) to be extracted by a shape detection algorithm.

This paper is organized as follows. After discussing previous work of Othmani et al. ([7]) in section II, we present the developed enhancement in section III. Experimental results are explained in section IV. Conclusion and future work are finally presented in section $\mathrm{V}$.

\section{RELATED WORK}

The method developed by Othmani et al. ([7]) assumes that tree cross-sections are circular ([3]). This justifies the use of pattern recognition in the point cloud. Their work is divided into four main steps.

First the point cloud is classified into soil points and vegetation points. The second step consists in clustering points according to the surface they lie on, and extracting the tree stem clusters by filtering them with a pattern recognition algorithm. The third step creates a skeleton of each detected 
tree. Finally trees positions and $\mathrm{DBH}$ are calculated in the fourth step.

\section{A. Soil Extraction and DTM Generation}

Determining for each point whether it is a soil point or a vegetation point is used as a pretreatment filter in several studies ([8]). Indeed, this classification allows further algorithms to focus on on the vegetation points for tree detection, and thus speeds up the chain of processes. Based on soil points a DTM is generated, giving a base reference for calculating the height at which DBH should be measured and total tree height.

The classification is done by calculating the minimum height in each cell of a horizontal regular grid. Then the density of points between the minimum height and $32 \mathrm{~cm}$ above is calculated and grid cells having a low density are filtered. The smoothness of the terrain is also analysed for filtering grid cells. Each cell containing in its neighbourhood a minimum height forming a zenithal angle with it lower than 45 degrees are eliminated. A digital terrain model (DTM) is generated from the previous grid by interpolating the minimum heights with a Delaunay triangulation. Interpolated values are considered to be the base height of the terrain. Because of the potential presence of low plants and noise and the possible variations of soil height in a cell of the horizontal grid, the points up to $32 \mathrm{~cm}$ from the base height are classified as soil points whereas other points are classified as vegetation.

\section{B. Clustering and Circle Fitting}

In this step, points belonging to the same tree stem are clustered. A circle will then be fitted to each cluster in order to filter non-stem clusters.

The cloud of vegetation points is sliced into $1 \mathrm{~cm}$ thick horizontal layers. In each layer, points are clustered according to their proximity: two points are clustered together if their are close enough one from another. Othmani et al. ([7]) used a FARO Focus3D terrestrial laser scanner with a scanning resolution of 0.036 degrees. They usually work on a $20 \mathrm{~m}$ radius circular forest plot. Spacing between two consecutive beams is around $1.2 \mathrm{~cm}$ at $20 \mathrm{~m}$. According to this value, and to overcome some occlusion issues, the clustering distance was fixed to $3 \mathrm{~cm}$. Clusters containing too few points to be representative are filtered.

A circle is then fitted to each cluster by a least square routine. Clusters leading to a circle fitting error above a threshold are filtered. Because tree stems are assumed to have a vertical continuity, vertical alignment of the fitted circle is used as an additional filter. For each circle $C$ detected, if no circle with a similar radius (empirically between $\frac{2}{3}$ and $\frac{3}{2}$ times the radius of $C$ ) located in the 50 upper and lower layers has its center in $C$, then the circle it is filtered.

At the end of this step only the clusters of points having a high degree of circularity and vertical coherence, and thus describing a tree stem, should remain.

\section{Skeletonization}

Obtaining the skeleton of a natural tree is an important issue that can be achieved in different ways ([9],[10]). In the work of Ohtmani et al. ([7]), this is done by aggregating tree stem clusters into virtual tree stems. First virtual sections are created according to vertical alignment of the circles. Virtual sections are then merged into virtual stems from which a skeleton is extracted.

Two clusters are affected to the same virtual section if their bounding box intersect horizontally and the difference between their height is lower than $50 \mathrm{~cm}$. For each virtual section, a skeleton is computed. Virtual sections are finally merged into virtual stems by analyzing their vertical extend and the alignment of their skeleton extremities. After merging the sections, the final skeleton of each virtual stem is computed and smoothed with a mobile-mean routine.

\section{Extraction of Tree's Attributes}

For each virtual stem, a circle is fitted to the stem points in a horizontal layer $1.30 \mathrm{~m}$ above the base height. The tree location is considered to be the center of this circle. Then, a serie of circles is fitted to the virtual tree stem points perpendicularly to the stem skeleton below $3.30 \mathrm{~m}$ from the DTM. For a better accuracy on the DBH measurement, it is deducted from the linear interpolation of the extracted diameters in which outliers are iteratively removed.

\section{METHODS AND MATERIALS}

The method presented above reaches its limits in the case of branch junctions of a tree as the hypothesis made on the tree cross-sections shape becomes invalid. Indeed, at branches junctions, cross-sections smoothly deform into a two circles shape. Fitting a single circle in this case leads to inaccurate measurements of stem position and diameter (results often become aberrant) and thus to errors while calculating stem taper. We therefore decided to introduce a new tree crosssection model, shifting from circles to arcs of circle.

We developed a two steps method in order to extract arcs of circle from three dimensional point clusters. First, we simplify each point cluster previously classified as tree stem. Reducing the amount of data allows an efficient use of further analysis and homogenizes the points cloud resolution. Another major effect of simplification is the smoothing of data, facilitating shape extraction. In the second step, each simplified cluster is analysed and those which are not classified as arc of circle are filtered.

\section{A. Clusters Simplification}

In this step, each cluster containing a sufficient amount of points is simplified. We adopted a simplification algorithm based on particle simulation inspired by Turk ([11]). This choice was motivated by its adaptability to point clouds as well as its flexibility, and the possible adaptation of the level of simplification to local surface features. The first main issue consists in finding the number of particles to be spread over the cluster. These particles are then randomly positioned in the three dimensional space. Then, an iterative process equalises their position over the surface using a point repulsion step followed by a relaxing step.

Each particle spread will repulse others in its neighbourhood so they won't aggregate but will disperse in the point cluster uniformly. This so-called repulsion step ensures an 
equalised description of the surface. We use the same linear repulsion force as in [12] and [11]. The force vectors are computed as:

$$
F_{i}(p)=k\left(r-\left\|p-p_{i}\right\|\right) \cdot\left(p-p_{i}\right)
$$

where $F_{i}(p)$ is the repulsive force exerted on particle $p$ by particle $p_{i}, k$ is a force constant and $r$ is the repulsion radius. At each iteration, the total repulsion applied to a particle $p$ is given by

$$
F(p)=\sum_{i \in N_{p}} F_{i}(p)
$$

where $N_{p}$ is the set of spread particles at distance less than $r$ from $p$.

After each repulsion step, a relaxation stage takes back the particles on the described surface. In our study, for the resulting simplified point cluster to be a subset of the original data, the relaxation is done by positioning every particles at the nearest point of the centroid of its neighbours.

The initial number of particles spread into each cluster is chosen to homogenize the resulting resolution. Otherwise, in order to get rid of the influence of density variations according to the distance to the scanner, this initial number is computed as a function of the bounding box of the cluster :

$$
\begin{aligned}
& \text { if }|\mathcal{C}| \geq n_{\text {min }} \text { and } \frac{p}{2 \cdot d} \geq n_{\text {min }} \\
& \qquad n=\frac{p}{2 \cdot d} \\
& \text { else }
\end{aligned}
$$

no simplification

where $n$ is the number of particles to spread, $|\mathcal{C}|$ is the size of the cluster, $n_{\min }$ is the minimal resulting number of particles set by the user, $p$ is the perimeter of the bounding box of the cluster to simplify, and $d$ is the particle spacing in the ideal case of a perfect and noiseless arc of circle. $n_{\min }$ has to be set according to the minimum number of points needed to describe a recognizable arc of circle. The ideal distance between two particles is set to the distance between two consecutive beams at the plot extremity. This ensure a homogenized sampling density.

\section{B. Shape Analysis}

Once clusters are simplified, they are analysed and filtered by a pattern recognition algorithm. Clusters are filtered if they do not fit a discrete arc of circle. The arc of circle detection we use was presented in [13]. Since this algorithm needs an ordinated set of points as input data, we first create, as a pretreatment, such polylines.

In order to create a polyline from a point cluster, a starting point is selected randomly as the polyline initialisation. The closest point to the extrema of the polyline is then added at the corresponding position and becomes the new extrema. This process is repeated until all the points in the cluster have been added to the polyline.
Each polyline is then transformed in the tangent space described in [13]. Let $C=\left\{C_{i}\right\}_{i=0}^{n}$ be a polyline, where $\left\{C_{i}\right\}_{i=0}^{n}$ are the $n$ points composing the line. Let $\alpha_{i}=\measuredangle\left(\overrightarrow{C_{i-1} C_{i}}, \overrightarrow{C_{i} C_{i+1}}\right)$ and $l_{i}=\left|C_{i} C_{i+1}\right|$. The following transformation maps the polyline onto the tangent space by creating segments $\left[T_{i 2} T_{(i+1) 1}\right]$ and $\left[T_{(i+1) 1} T_{(i+1) 2}\right]$ for $i \in[0, n-1]$

$$
\begin{aligned}
& T_{02}=(0,0) \\
& T_{i 1}=\left(T_{(i-1) 2} \cdot x+l_{i-1}, T_{(i-1) 2} . y\right) \quad \forall i \in[1, n] \\
& T_{i 2}=\left(T_{i 1} \cdot x, T_{i 1} . y+\alpha_{i}\right) \quad \forall i \in[1, n-1]
\end{aligned}
$$

Arcs of circle show interesting properties in this tangent space. Polylines can be considered as arcs of circle if, and only if:

$$
\begin{aligned}
& \sin \alpha_{i} \simeq \alpha_{i} \\
& \frac{l_{1}+l_{2}}{\alpha_{1}} \simeq \frac{l_{2}+l_{3}}{\alpha_{2}} \simeq \cdots \simeq{\frac{l_{n-1}+l_{n}}{\alpha_{n-1}}}^{\forall i}
\end{aligned}
$$

Hence, in the tangent space, the mid-points of the segments $\left[T_{i 2} T_{(i+1) 1}\right]$ and $\left[T_{(i+1) 1} T_{(i+1) 2}\right]$ are aligned and form a fuzzy line ([13]). Thus, arc of circle recognition is reduced to a line detection problem.

Therefore, we use a simple linear regression to fit a line to the mid-points of the tangent space. The simplified point clusters leading to a residual error above a threshold are filtered. This value must be adapted according to the data set, especially the type of bark of the trees. A low value is sufficient for smooth barks while rough barks are more difficult to segment as an arc and needs a higher value. According to this, values between $20^{\circ}$ and $50^{\circ}$ represent a compromise between the quality of the filter and the number of false negatives.

\section{RESULTS}

In order to validate our approach, we inserted our filtering step in the tree extraction process described by Othmani et al. in [7] and compared the models obtained with and without the present filter. Figures (1) (a), (b) and (c) show the experimental results obtained on two individual trees extracted from natural forest plots. As can be seen, the initial methodology leads to aberrant cylinders at branching junctions as well as in branches and/or foliage merging areas. Filtering such data was the main objective of our filter as in such a setting, the resulting tree models can be unrealistic and lead to automatic measurement errors.

As shown in figure (1) (a), (b) and (c), inserting our filter in the reconstruction process efficiently reduces the number of aberrant cylinders and hence improves the accuracy of the final models and of automatic extractions (such as DBH extraction).

Our filter mostly operates at branching junctions and removes aberrant cylinders created by unwanted foliage data in horizontal clusters. However it may lead to the elimination of good clusters since the pattern recognition algorithm we use is more restrictive than the filtering in [7]. Let us mention that however, it does not fully filter any noise in the data.

The threshold on line fitting residual error in the tangent space for arc of circle recognition is a crucial parameter since it strongly influences the quality of results. Its value has to 

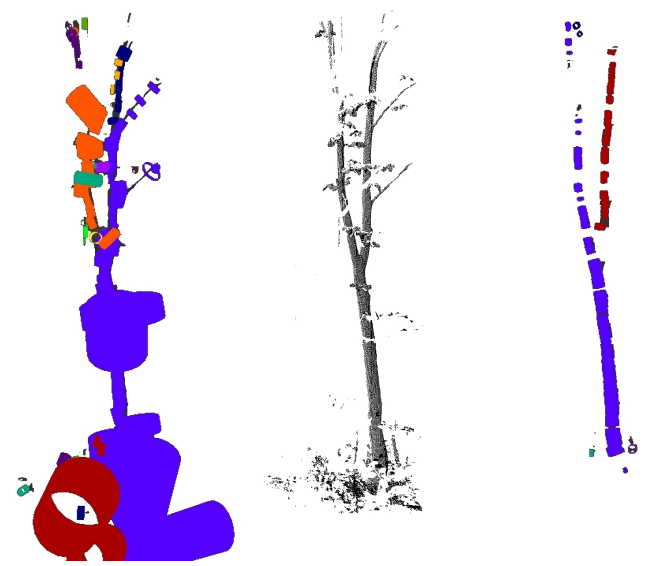

(a)
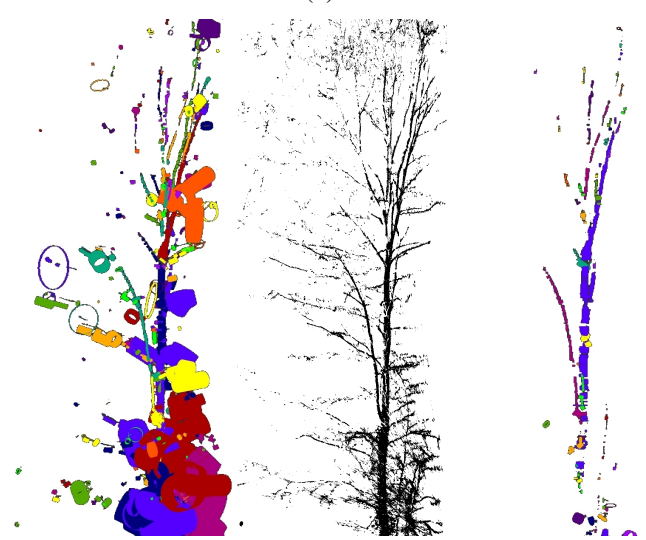

(b)

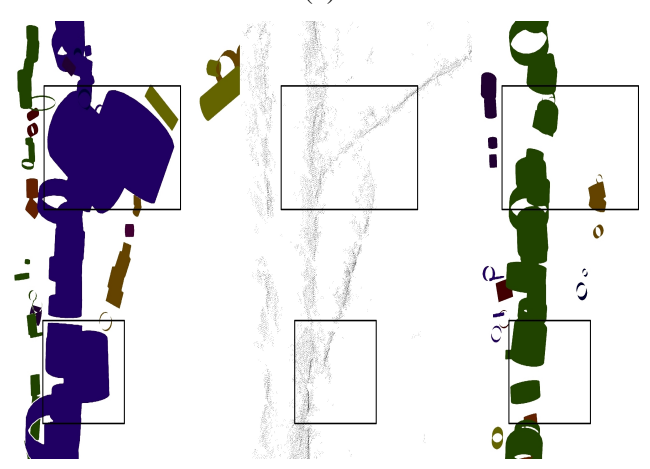

(c)

Fig. 1. Result of tree stem modeling - (left) Othmani results, (center) initial cloud, (right) Othmani reconstruction with our filter.

be set with precaution and might be slightly adjusted to the number of points contained by each analysed cluster in order to reduce statistical effects.

\section{CONCLUSION AND PERSPECTIVES}

In this paper we propose a two steps enhancement for tree stem reconstruction processes (in particular, we tested our approach by integrating it to [7]). Our work constitutes a novel combination and application of two point cloud processing algorithms: namely a particles simulation simplification and an arc of circles pattern recognition algorithm. This approach leads to an improvement of the automatic extraction of natural tree stems attributes based on circle fitting (such as in [7]).
Indeed, most aberrant cylinders are successfully filtered which highly improves constructed tree models. As mentioned earlier, such a digital modelling is a central issue in order to preserve, control and promote our forest and wooded areas heritage. But obviously, accuracy is the primary quality expected from such a digitalisation.

In further work, we intend to use the arc of circle detection to segment the clusters according to the branches position by segmenting each fuzzy line in the tangent space. Fuzzy line extraction will reduce the sensitivity to the choice of the threshold in the line fitting used in the present work. It will therefore lead to a better separation of branches at branching junctions and hence will allow the filter to keep stem points of a cluster while filtering foliage points it contains.

We also intend to improve our usage particle simulation simplification. On the one hand, it should better handle the local geometry of the objects. On the other hand, a simplification step producing smoother resulting point sets would help further pattern recognition.

\section{REFERENCES}

[1] M. Dassot, T. Constant, and M. Fournier, "The use of terrestrial lidar technology in forest science: application fields, benefits and challenges," Annals of Forest Science, vol. 68, no. 5, pp. 959-974, 2011.

[2] H.-G. Maas, A. Bienert, and al., "Automatic forest inventory parameter determination from terrestrial laser scanner data," International Journal of Remote Sensing, vol. 29, no. 5, pp. 1579-1593, 2008.

[3] M. Simonse, T. Aschoff, H. Spiecker, and M. Thies, "Automatic determination of forest inventory parameters using terrestrial laser scanning," in Proceedings of the ScandLaser Scientific Workshop on Airborne Laser Scanning of Forests, 2003, pp. 1-7.

[4] N. Pfeifer and D. Winterhalder, "Modelling of tree cross sections from terrestrial laser scanning data with free-form curves," ISPRS Archives, vol. 36, no. Part 8, pp. 76-81, 2004.

[5] P. Raumonen, M. Kaasalainen, M. kerblom, S. Kaasalainen, H. Kaartinen, M. Vastaranta, M. Holopainen, M. Disney, and P. Lewis, "Fast automatic precision tree models from terrestrial laser scanner data," Remote Sensing, vol. 5, no. 2, pp. 491-520, 2013.

[6] M. Béland, J.-L. Widlowski, R. Fournier, J.-F. Côté, and M. Verstraete, "Estimating leaf area distribution in savanna trees from terrestrial lidar measurements," in Agricultural and Forest Meteorology, Elsevier, Ed., vol. 151, 2011, pp. 1252-1266.

[7] A. Othmani, A. Piboule, M. Krebs, C. Stolz, and L. Lew Yan Voon, "Towards automated and operational forest inventories with t-lidar," in SilviLaser, 2011

[8] M. W. McDaniel, T. Nishihata, C. A. Brooks, P. Salesses, and K. Iagnemma, "Terrain classification and identification of tree stems using ground-based lidar," Journal of Field Robotics, vol. 29, no. 6, pp. 891910, 2012.

[9] A. Bucksch and R. Lindenbergh, "Campinoa skeletonization method for point cloud processing," ISPRS journal of photogrammetry and remote sensing, vol. 63, no. 1, pp. 115-127, 2008.

[10] A. Bucksch, R. Lindenbergh, and M. Menenti, "Skeltre," The Visual Computer, vol. 26, no. 10, pp. 1283-1300, 2010.

[11] G. Turk, "Re-tiling polygonal surfaces," ACM SIGGRAPH Computer Graphics, vol. 26, no. 2, pp. 55-64, 1992.

[12] M. Pauly, M. Gross, and L. Kobbelt, "Efficient simplification of pointsampled surfaces," in Proceedings of the conference on Visualization '02, 2002, pp. 163-170.

[13] T. P. Nguyen and I. Debled-Rennesson, "Arc segmentation in linear time," in Proceedings of the 14th international conference on Computer analysis of images and patterns - Volume Part I, ser. CAIP'11. Berlin, Heidelberg: Springer-Verlag, 2011, pp. 84-92. 